\title{
FIXED POINT THEORY AND THE $K$-THEORETIC TRACE
}

\author{
ROSS GEOGHEGAN \\ Department of Mathematical Sciences \\ Binghamton University \\ Binghamton, NY 13902-6000, USA \\ E-mail: ross@math.binghamton.edu \\ ANDREW NICAS \\ Department of Mathematics and Statistics \\ McMaster University \\ Hamilton, Ontario L8S 4K1, Canada \\ E-mail: nicas@mcmaster.ca
}

\begin{abstract}
The relationship between fixed point theory and $K$-theory is explained, both classical Nielsen theory (versus $K_{0}$ ) and 1-parameter fixed point theory (versus $K_{1}$ ). In particular, various zeta functions associated with suspension flows are shown to come in a natural way as "traces" of "torsions" of Whitehead and Reidemeister type.
\end{abstract}

1. Introduction. Consider the following facts:

1. In modern geometric topology, $K$-theoretic obstructions to achieving desirable geometric goals sometimes occur. Examples include the Wall finiteness obstruction [Wa], the Whitehead torsion of an $h$-cobordism $[\mathrm{C}],[\mathrm{RS}]$ and $[\mathrm{K}]$, and the various Reidemeister torsions associated with a suspension flow $[\mathrm{M}],[\mathrm{F}],\left[\mathrm{GN}_{2}\right]$.

2. In recent papers $\left[\mathrm{GN}_{1-4}\right]$ we have explained why $n$-parameter Nielsen Fixed Point Theory is obstructed by an element of an $n^{\text {th }}$ Hochschild homology group. [For $n=0$, this says nothing new as the $0^{\text {th }}$ Hochschild homology group is naturally isomorphic to a familiar free abelian group employed in Nielsen theory.]

1991 Mathematics Subject Classification: Primary 55M20; Secondary 19D55, 57R80, 58F20.

The first author is partially supported by the National Science Foundation. The second author is partially supported by the Natural Sciences and Engineering Research Council of Canada.

The paper is in final form and no version of it will be published elsewhere. 
3. For any associative ring $S$ with unit, there is a $K$-theoretic trace:

$$
\operatorname{Tr}_{*}: K_{*}(S) \rightarrow H H_{*}(S)
$$

from the $K$-theory of $S$ to the Hochschild homology of $S$.

4. It sometimes happens that a geometric problem is obstructed by an element $\alpha$ in some $K$-group, while an obviously related fixed point problem is obstructed by the Hochschild homology element $\operatorname{Tr}_{*}(\alpha)$.

5. It sometimes happens that a fixed point problem is obstructed by a Hochschild homology element $\beta$ which is not in the image of the trace $\operatorname{Tr}_{*}$.

6. On the other hand, it sometimes happens that $\beta$ lies in the image of the trace and in this manner one is led to an interesting $K$-theoretic obstruction which was not previously evident.

We lay out these facts in this paper in some detail. Examples of Fact 4 are given in $\S 2$ and $\S 3$, of Fact 5 in $\S 2$ and $\S 5$, and of Fact 6 in $\S 4$. While the necessary theorems have already appeared in $\left[\mathrm{G}_{1}\right]$ and $\left[\mathrm{GN}_{1-4}\right]$, they have not been previously presented from the point of view expressed here. This paper is an expanded version of the talk given by one of us (Geoghegan) at the Warsaw meeting.

2. Classical Nielsen theory and $K_{0}$. Let $f:(K, v) \rightarrow(K, v)$ be a pointed cellular map on a finite CW complex, inducing $\phi: G \rightarrow G$ on fundamental groups. Let $G_{\phi}$ be the set of semiconjugacy classes with respect to $\phi$, where $g_{1}, g_{2} \in G$ are semiconjugate if there exists $g \in G$ with $g_{1}=g g_{2} \phi(g)^{-1}$. The Nielsen theory of $f$ is encoded by the Reidemeister trace $R(f, v) \in \mathbb{Z} G_{\phi}$, the free abelian group generated by $G_{\phi}$. The geometric definition of $R(f, v)$ is:

$$
R(f, v)=\sum_{\text {fixed point classes } \mathbf{F}} \iota(f, \mathbf{F}) \Phi(\mathbf{F}) \in \mathbb{Z} G_{\phi}
$$

where $\Phi$ is the well-known injection of fixed point classes to semiconjugacy classes, and $\iota(f, \mathbf{F}) \in \mathbb{Z}$ is the index of $f$ at the fixed point class $\mathbf{F}$ (see $[\mathrm{B}]$ or $\left[\mathrm{GN}_{1}, \S 1(\mathrm{~B})\right]$ ). Choose an orientation for each cell of $K$. Let $(\tilde{K}, \tilde{v})$ be the universal cover of $(K, v)$, orient the cells of $\tilde{K}$ compatibly with those of $K$, choose one cell in each $G$-orbit of cells to get a free $\mathbb{Z} G$-basis for the cellular chains $C_{*}(\tilde{K})$. The algebraic definition of $R(f, v)$ is:

$$
R(f, v)=\sum_{k \geq 0}(-1)^{k} q\left(\operatorname{trace}\left(\tilde{f}_{k}: C_{k}(\tilde{K}) \rightarrow C_{k}(\tilde{K})\right)\right) \in \mathbb{Z} G_{\phi}
$$

where $q: \mathbb{Z} G \rightarrow \mathbb{Z} G_{\phi}$ extends the function which sends each $g \in G$ to its semiconjugacy class (see $\left[\mathrm{GN}_{1}, \S 1(\mathrm{~B})\right]$ ). Here, $C_{*}(\tilde{K})$ is regarded as a right $\mathbb{Z} G$ module, and $\tilde{f}_{k}$ denotes the $\mathbb{Z} G$-matrix whose $(i, j)^{\text {th }}$ entry is the coefficient of the $i^{\text {th }} k$-cell in the image of the $j^{\text {th }} k$-cell (considered as basis elements); see [ $\left.\mathrm{GN}_{1}, \S 1(\mathrm{~B})\right]$.

The assertion that these two definitions are equivalent is essentially due to Reidemeister $[\mathrm{R}]$ and Wecken $[\mathrm{W}]$; see, for example, $\left[\mathrm{G}_{2}\right]$ for a proof.

In this section we will assume that $\phi$ is the identity endomorphism $1: G \rightarrow G$, and $G_{1}$ will denote the set of conjugacy classes of $G$. So $R(f, v) \in \mathbb{Z} G_{1}$. We recall the definition of $K_{0}(\mathbb{Z} G)$ and $\tilde{K}_{0}(\mathbb{Z} G)$. Denote the isomorphism class of a finitely generated projective $\mathbb{Z} G$-module $P$ by $[P]$. The abelian group $K_{0}(\mathbb{Z} G)$ is defined to be the free abelian group 
generated by all such $[P]$ modulo the relations $[P]+[Q]=[P \oplus Q]$. Let $\{P\}$ be the corresponding element of $K_{0}(\mathbb{Z} G)$. There is a natural homomorphism $\mathbb{Z} \rightarrow K_{0}(\mathbb{Z} G)$ taking 1 to $\{\mathbb{Z} G\}$. The cokernel, $\tilde{K}_{0}(\mathbb{Z} G)$, is the reduced projective class group of the ring $\mathbb{Z} G$. We write $\pi: K_{0}(\mathbb{Z} G) \rightarrow \tilde{K}_{0}(\mathbb{Z} G)$ for the quotient homomorphism. For us, the only relevant property of $\tilde{K}_{0}(\mathbb{Z} G)$ is distinguishing zero from non-zero elements: $\pi(\{P\})=0$ if and only if $P$ is stably free, i.ethe direct sum of $P$ with some finitely generated free module is a free module. See, for example, [Br, p.201].

If $A$ is an idempotent $m \times m \mathbb{Z} G$-matrix, the image of $A: \mathbb{Z} G^{m} \rightarrow \mathbb{Z} G^{m}$ is a projective $\mathbb{Z} G$-module; conversely if $P$ is a finitely generated projective $\mathbb{Z} G$-module it is a direct summand of a free module $\mathbb{Z} G^{m}$, and the projection of $\mathbb{Z} G^{m}$ onto the submodule $P$ of $\mathbb{Z} G^{m}$ is represented by an idempotent matrix. So elements of $K_{0}(\mathbb{Z} G)$ can be regarded as equivalence classes $\{A\}$ of idempotent $\mathbb{Z} G$-matrices $A$. The homomorphism $A \mapsto \operatorname{trace}(A) \mapsto q(\operatorname{trace}(A))$ well-defines the $K$-theoretic (or Hattori-Stallings) trace $\operatorname{Tr}_{0}: K_{0}(\mathbb{Z} G) \rightarrow \mathbb{Z} G_{1}$. For a proof that this is well-defined see [St, $\left.\S 1\right]$.

When is $R(f, v)$ in the image of $\operatorname{Tr}_{0}$ ? A partial answer is: When $f$ is a homotopy idempotent, i.e. $f$ is homotopic to $f^{2}$, as we now explain.

Let $f$ be a homotopy idempotent. We say that $f$ splits if there exist a (not necessarily finite) CW complex $X$ and maps $d: K \rightarrow X$ and $u: X \rightarrow K$ such that $d \circ u \simeq \operatorname{id}_{X}$ and $u \circ d \simeq f$. In that case $X$ is a finitely dominated complex (the existence of $K, u$ and $d$ with $K$ finite and $d \circ u \simeq \operatorname{id}_{X}$ being the definition). We may assume (giving $X$ a basepoint) that $u$ and $d$ are basepoint preserving maps. It is a straightforward theorem that if $f \simeq f^{2}$ rel $v$ then $f$ splits. A much harder theorem of Hastings and Heller asserts that the "rel $v$ " is unnecessary:

THeOREM [HH]. If $f$ is a homotopy idempotent on a finite dimensional $C W$ complex $K$ then $f$ splits.

For another proof see [BG].

So a homotopy idempotent $f:(K, v) \rightarrow(K, v)$ always gives rise in this way to a finitely dominated complex $X$; indeed $X$ is unique up to homotopy equivalence. Conversely, given $X$, a homotopy idempotent $f=u \circ d:(K, v) \rightarrow(K, v)$ can always be chosen which splits through $X$ and satisfies $f_{\#}=1: G \rightarrow G$. Finally, if $f^{\prime}:\left(K^{\prime}, v^{\prime}\right) \rightarrow\left(K^{\prime}, v^{\prime}\right)$ is another homotopy idempotent splitting through $X$ then $R(f, v)$ and $R\left(f^{\prime}, v^{\prime}\right)$ encode the same information (same Nielsen numbers, same indices, canonically corresponding fixed point classes). For more on all this, see $\left[\mathrm{G}_{1}\right]$.

In view of what has been said, we will assume in what follows that $u$ and $d$ induce isomorphisms on fundamental groups and we will identify $\pi_{1}(K, v)$ with $\pi_{1}(X, x)$ via $d_{\#}$, calling both groups $G$.

Next, we discuss the Wall obstruction $\sigma(X) \in \tilde{K}_{0}(\mathbb{Z} G)$ of a finitely dominated space $X$ whose fundamental group is $G$. This is defined in [Wa]; for a helpful relevant discussion, see [Br, ch. 8], especially page 201.

TheOREM [Wa]. $\sigma(X)=0$ if and only if $X$ has the homotopy type of a finite complex.

Choose $A$ so that $\pi(\{A\})=\sigma(X)$, and consider $\operatorname{Tr}_{0}(\{A\}) \in \mathbb{Z} G_{1}$. If $I$ is a $k \times k$ identity matrix then $\pi(\{A\})=\pi(\{A \oplus I\})$ and $\operatorname{Tr}_{0}(\{A \oplus I\})=\operatorname{Tr}_{0}(\{A\})+k[1]$, so the 
sum of the coefficients in $\operatorname{Tr}_{0}(\{A\})$ is not determined by $\sigma(X)$, but can be any integer. The following theorem is essentially proved in $\left[\mathrm{G}_{1}\right]$ :

ThEOREM 2.1. If $\pi(\{A\})=\sigma(X)$ and $A$ is chosen so that the sum of the coefficients in $\operatorname{Tr}_{0}(\{A\})$ is the Euler characteristic $\chi(X)$ then $R(f, v)=\operatorname{Tr}_{0}(\{A\}) \equiv q(\operatorname{trace}(A))$.

In summary, the Reidemeister trace of a homotopy idempotent on a finite complex always lies in the image of the $K$-theoretic trace $\operatorname{Tr}_{0}: K_{0}(\mathbb{Z} G) \rightarrow \mathbb{Z} G_{1}$.

The Strong Bass Conjecture [Ba] asserts that the image of this trace is always a multiple of the trivial conjugacy class $[1] \in G_{1} \subset \mathbb{Z} G_{1}$. This conjecture is known to be valid for some large classes of groups (see $\left[\mathrm{G}_{2}\right]$ for references). When this holds for $G$, it follows that for any map $f:(K, v) \rightarrow(K, v)$ (not necessarily a homotopy idempotent) inducing the identity on the fundamental group $G, R(f, v)$ is in the image of the trace if and only if the Nielsen number $N(f)=0$ or 1 and when $N(f)=1$ the only essential fixed point class is that of the basepoint.

When $f$ does not induce the identity on the fundamental group (and is not "equivalent" to a map $g$ which does, i.e. $f=r \circ s$ and $g=s \circ r$ ) it is not clear to us if $K_{0}$ of some other ring might replace $K_{0}(\mathbb{Z} G)$ giving $R(f, v)$ in the image of some sort of $K$-theoretic trace.

Finally, we remark that from an algebraic point of view, it is useful to view $\mathbb{Z} G_{1}$ as the $0^{\text {th }}$ Hochschild homology group $H H_{0}(\mathbb{Z} G)$ of the ring $\mathbb{Z} G$. This could be avoided up to now, but must be confronted in the next section.

\section{The $s$-Cobordism Theorem and a related one-parameter fixed point}

problem. In this section we recall 1-parameter Nielsen theory and the $s$-Cobordism Theorem, and we relate the two via the $K$-theoretic trace.

3(A). One-parameter Nielsen fixed point theory. Let $F: M \times I \rightarrow M$ be a homotopy where $M$ is a compact oriented manifold. We consistently work in the smooth or PL category. Pick a basepoint $v \in M$. Identify $\pi_{1}(M \times I,(v, 0))$ with $G \equiv \pi_{1}(M, v)$ using the isomorphism induced by the projection map $p: M \times I \rightarrow M$. Define $\operatorname{Fix}(F)=\{(x, t) \in$ $M \times I \mid F(x, t)=x\}$; its elements are the fixed points of $F$.

Assume $F(v, 0)=v$ and that $F$ induces the identity $1: G \rightarrow G$. Two fixed points $(x, t)$ and $\left(y, t^{\prime}\right)$ of $F$ are in the same fixed point class if for some path $\nu$ from $(x, t)$ to $\left(y, t^{\prime}\right)$, the loop $(p \circ \nu)(F \circ \nu)^{-1}$ is homotopically trivial. This defines an equivalence relation on $\operatorname{Fix}(F)$. Just as in the classical case, there is an injective function $\Psi$ from the set of fixed point classes of $F$ to the set, $G_{1}$, of conjugacy classes: the class containing $(x, t)$ is mapped to the conjugacy class $C$ containing $g_{C} \equiv\left[(p \circ \mu)(F \circ \mu)^{-1}\right]$, where $\mu$ is any path from the basepoint $(v, 0)$ to $(x, t)$. It is easy to check that $\Psi$ is well-defined and injective, that $F$ has only finitely many fixed point classes, and that fixed points in the same path component of $\operatorname{Fix}(F)$ are in the same fixed point class. Moreover, with the same notation, let $\omega$ be a loop in $\operatorname{Fix}(F) \subset M \times I$ based at $(x, t)$, and let $h=\left[\mu \omega \mu^{-1}\right] \in G$; then $h$ lies in the centralizer, $Z\left(g_{C}\right)$, of $g_{C}$.

By transversality, we can perturb $F$ rel $M \times\{0,1\}$ so that $\operatorname{Fix}(F)$ consists of transverse circles in the interior of $M \times I$ which, for all but finitely many values of $t$, cross $M \times\{t\}$ 
transversely, and other path components of fixed points each of which meets $M \times\{0,1\}$. If $(x, t)$ lies in such a circle crossing $M \times\{t\}$ transversely, orient the circle in the direction of positive time if $\iota\left(F_{t}, x\right)$ is positive, and in the other direction if it is negative; see $[\mathrm{DG}, \S 8$ and $\S 11]$ for details.

We are only interested in the circles, indeed in circles not in the same fixed point class as any fixed points in $M \times\{0,1\} .{ }^{1}$ Let $V$ be such a circle, let $(x, t) \in V$, let $\mu$ be a path in $M \times I$ from $(v, 0)$ to $(x, t)$, and let $\omega$ be the loop based at $(x, t)$ obtained by traversing $V$ once in the direction of its orientation. With notation as above, we have, as previously indicated, that the corresponding element, $h$, of $G$ lies in $Z\left(g_{C}\right)$. In this way, we associate with $V$ an element of $H_{1}\left(Z\left(g_{C}\right)\right) \cong\left(Z\left(g_{C}\right)\right)_{\text {ab. }}$. If there are two circles $V_{1}$ and $V_{2}$ in the same fixed point class, we reach the same centralizer $Z\left(g_{C}\right)$ from both circles provided the path used for $\left(x_{1}, t_{1}\right) \in V_{1}$ is $\mu$, and the path used for $\left(x_{2}, t_{2}\right) \in V_{2}$ is $\mu \nu$, where $p(\nu) F(\nu)^{-1}$ is homotopically trivial; we treat any (finite) number of circles similarly. Thus, for each fixed point class, $\mathbf{F}$, of $F$ which does not meet $M \times\{0,1\}$, we have defined an element $\iota(F, \mathbf{F}) \in H_{1}\left(Z\left(g_{C}\right)\right)$, where $g_{C} \equiv\left[p(\mu) F(\mu)^{-1}\right]$ represents the conjugacy class $C$, and $\Psi(\mathbf{F})=C$.

The transverse intersection invariant of $F$ is:

$$
R(F, v)=\sum_{j=1}^{k} \iota\left(F, \mathbf{F}_{j}\right) \in \bigoplus_{C \in G_{1}} H_{1}\left(Z\left(g_{C}\right)\right)
$$

where $\mathbf{F}_{1}, \ldots, \mathbf{F}_{k}$ are the fixed point classes of $F$ which do not meet $M \times\{0,1\}$. Note that since $F_{0}(v)=v$, the fixed point class of $v$ is not among the $\mathbf{F}_{j}$ 's.

Now, $\bigoplus_{C \in G_{1}} H_{1}\left(Z\left(g_{C}\right)\right)$ should remind the reader of

$$
\bigoplus_{C \in G_{1}} H_{0}\left(Z\left(g_{C}\right)\right) \cong \bigoplus_{C \in G_{1}} \mathbb{Z} \cong \mathbb{Z} G_{1}
$$

in $\S 2$; these direct sums have the following algebraic reformulation.

The Hochschild homology groups of an associative ring with unit, $S$, are the homology groups of a certain chain complex which in degrees 2, 1, 0 is given by:

$$
\begin{aligned}
& \cdots \longrightarrow S \otimes S \otimes S \stackrel{d_{2}}{\longrightarrow} S \otimes S \stackrel{d_{1}}{\longrightarrow} S \\
& s_{1} \otimes s_{2} \otimes s_{3} \mapsto \quad s_{2} \otimes s_{3} s_{1}-s_{1} s_{2} \otimes s_{3}+s_{1} \otimes s_{2} s_{3} \\
& s_{1} \otimes s_{2} \mapsto s_{2} s_{1}-s_{1} s_{2} .
\end{aligned}
$$

In particular, $H H_{0}(S)=\operatorname{coker}\left(d_{1}\right)$ and $H H_{1}(S)=\operatorname{ker}\left(d_{1}\right) / \operatorname{image}\left(d_{2}\right)$. When $S=\mathbb{Z} G$ there are isomorphisms $H H_{i}(\mathbb{Z} G) \cong \bigoplus_{C \in G_{1}} H_{i}\left(Z\left(g_{C}\right)\right.$ ) for all $i \geq 0$ (see $\left[\mathrm{GN}_{1-4}\right.$ ] for a detailed description). These isomorphisms are canonical once the representatives $g_{C}$ of the conjugacy classes $C$ have been chosen. This suggests that it may be useful to regard $R(F, v)$ as an element of $H H_{1}(\mathbb{Z} G)$.

3(B). $K_{1}$, the Whitehead group, and Whitehead torsion. Let $S$ be a ring with unit, let $G L(n, S)$ denote the general linear group consisting of all $n \times n$ invertible matrices

\footnotetext{
1 The reason for this is that in the remaining fixed point classes the distinction between circles and other fixed points is not invariant under homotopy rel $M \times\{0,1\}$. For example, a transverse arc of fixed points beginning and ending in $M \times\{0,1\}$ might merge with a transverse circle in the same fixed point class after a homotopy rel $M \times\{0,1\}$.
} 
over $S$, and let $G L(S)$ be the direct limit of the sequence $G L(1, S) \subset G L(2, S) \subset \cdots$ A matrix in $G L(S)$ is called elementary if it coincides with the identity except for a single off-diagonal entry. The subgroup $E(S) \subset G L(S)$ generated by the elementary matrices is precisely the commutator subgroup of $G L(S)$ (see [C]) and the abelian quotient group $G L(S) / E(S)$ is, by definition, $K_{1}(S)$. If $A \in G L_{n}(S)$ the image of $A$ in $K_{1}(S)$ is called the torsion of $A$.

Now let $U \subset S^{*}$ be a subgroup of the group of units of $S$. Assume $S$ has the property that any two bases of a finitely generated free module have the same cardinality. Declare two bases of a finitely generated free module to be equivalent if the change of basis matrix represents an element of $K_{1}(S)$ which lies in the image of the natural map $U \rightarrow K_{1}(S)$. Define $K_{1}^{U}(S) \equiv \operatorname{coker}\left(U \rightarrow K_{1}(S)\right)$. Let $(C, \partial)$ be a finitely generated chain complex of right modules over $S$ such that each $C_{i}$ is free with a given equivalence class of bases. Suppose that $C$ is acyclic. Let $\delta: C \rightarrow C$ be a chain contraction. Define $C_{\text {odd }} \equiv \bigoplus_{\text {iodd }} C_{i}$ and $C_{\text {even }} \equiv \bigoplus_{i \text { even }} C_{i}$. The restriction of $\partial+\delta$ to $C_{\text {odd }}$ is an isomorphism $C_{\text {odd }} \rightarrow C_{\text {even }}$ and so its matrix with respect to bases chosen from each of the given equivalence classes defines an element of $K_{1}(S)$. The image of this element in $K_{1}^{U}(S)$ is independent of the choice of representatives of the equivalence classes of bases (see [C]); it is called the torsion of $(C, \partial)$ with respect to $U$. The important case for the purpose of this section is $S=\mathbb{Z} G$ and $U= \pm G$. In this case $\mathrm{Wh}(G) \equiv K_{1}^{ \pm G}(\mathbb{Z} G)$ is called the Whitehead group of $G$ and the torsion of an acyclic complex $(C, \partial)$ as above is called its Whitehead torsion.

An $h$-cobordism $\left(M ; M_{0}, M_{1}\right)$ is a manifold $M$ with two boundary components $M_{0}$ and $M_{1}$ both of which are strong deformation retracts of $M$. The torsion of $\left(M ; M_{0}, M_{1}\right)$ is the Whitehead torsion, $\tau(M)$, of the relative cellular chain complex $C_{*}\left(\tilde{M}, \tilde{M}_{0}\right)$ with respect to a $\mathrm{CW}$ structure on the $\operatorname{triad}\left(M ; M_{0}, M_{1}\right)$. In $[\mathrm{RS}]$ one finds a proof of:

TheOrem ( $s$-Cobordism Theorem). If $\operatorname{dim} M \geq 6$ and $M$ is compact, $\left(M ; M_{0}, M_{1}\right)$ is $P L$ homeomorphic to $\left(M_{0} \times I, M_{0} \times\{0\}, M_{0} \times\{1\}\right)$ if and only if $\tau(M)=0$.

The same theorem is true in the smooth category, see $[\mathrm{K}]$.

3(C). The $K$-theoretic trace. The $K$-theoretic trace (or Dennis trace) $\operatorname{Tr}_{1}: K_{1}(S) \rightarrow$ $H H_{1}(S)$ is defined as follows. If $\alpha \in K_{1}(S)$ is represented by an invertible $n \times n$ matrix $A$, then $\operatorname{Tr}_{1}(\alpha)$ is represented by the Hochschild 1-cycle $\sum_{i, j} A_{i j} \otimes\left(A^{-1}\right)_{j i}$.

Consider the diagram

$$
\begin{aligned}
& K_{1}(\mathbb{Z} G) \stackrel{\operatorname{Tr}_{1}}{\longrightarrow} \quad H H_{1}(\mathbb{Z} G) \cong \bigoplus_{C \in G_{1}} H_{1}\left(Z\left(g_{C}\right)\right) \\
& \operatorname{Wh}(G)
\end{aligned}
$$

where $\pi$ is the obvious quotient. The feature to note is:

Proposition 3.1. If $\alpha, \beta \in K_{1}(\mathbb{Z} G)$ are such that $\pi(\alpha)=\pi(\beta)$ then $\operatorname{Tr}_{1}(\alpha)-\operatorname{Tr}_{1}(\beta)$ can only be non-zero in the component of the trivial conjugacy class $[1] \in G_{1}$.

3(D). The $K$-theoretic trace and Nielsen fixed point theory. Let $\left(M ; M_{0}, M_{1}\right)$ be an $h$-cobordism with $M$ compact. Let $F: M \times I \rightarrow M$ be a strong deformation retract of $M$ onto $M_{0}$. Then we can perturb $F$ rel $M \times\{0,1\}$ (using relative transversality and ad hoc methods) so that $\operatorname{Fix}(F)=(M \times\{0\}) \cup\left(M_{0} \times I\right) \cup$ transverse circles as described in 
(B). Now, $(M \times\{0\}) \cup\left(M_{0} \times I\right)$ as well as, perhaps, some circles are in the fixed point class of the basepoint $v$. We are only interested in the other circles, namely the ones that make up the components of $R(F, v)$. Those are the ones that cannot disappear under perturbation of $F \operatorname{rel} M \times\{0,1\}$.

Here is the main theorem of this section.

TheOREM 3.2. If $\pi(\alpha)=\tau(M)$ then the projection of $\operatorname{Tr}_{1}(\alpha)$ away from the component of the trivial conjugacy class is equal to $R(F, v)$.

In other words, apart from an ambiguity over the trivial conjugacy class (analogous to the ambiguity in getting $\{A\}$ from $\sigma(X)$ in Theorem 2.1), the one-parameter fixed point problem contained in our setup is the "trace problem" associated with the $h$-cobordism problem. Note that if $M$ is homeomorphic to $M_{0} \times I$ and if $F$ is the "projection" (i.e. $F((y, u), t)=(y, u t)$ where $\left.(y, u) \in M_{0} \times I\right)$ then $\operatorname{Fix}(F)=M \times\{0\} \cup M_{0} \times I$, so $R(F, v)=0$.

Proposition 3.1 and Theorem 3.2 are proved in $\left[\mathrm{GN}_{1}, \S 7\right]$.

4. Torsion and zeta functions. Let $f:(K, v) \rightarrow(K, v)$ be a pointed cellular map on a finite CW complex, inducing $\phi: G \rightarrow G$ on fundamental groups. In this section we do not assume that $\phi$ is the identity. A periodic point of period $m$ is a fixed point of the $m$-fold iterate $f^{m}$. If $\mathbf{F}$ is a fixed point class of the map $f^{m}$, so are $f(\mathbf{F}), \ldots, f^{m-1}(\mathbf{F})$, and the corresponding $m$-periodic orbit class of $f$ is $\bigcup_{j=0}^{m-1} f^{j}(\mathbf{F})$, where $f^{0}(\mathbf{F}) \equiv \mathbf{F}$. Note that the fixed point classes $f^{i}(\mathbf{F})$ and $f^{j}(\mathbf{F})$ are either identical or disjoint.

One classifies $m$-periodic orbit classes algebraically thus: the relation $g \sim \phi(g)$ welldefines an equivalence relation on the set $G_{\phi^{m}}$; write $G_{\phi^{m}} /\langle\phi\rangle$ for the set of equivalence classes. By analogy with $\Phi$ in $\S 2$, there is an injective function $\Phi^{\prime}$ from the set of $m$ periodic orbit classes of $f$ to the set $G_{\phi^{m}} /\langle\phi\rangle$. Define

$$
\bar{R}\left(f^{m}, v\right)=\sum_{\begin{array}{c}
m \text {-periodic } \\
\text { orbit classes } \mathbf{O}
\end{array}} \iota\left(f^{m}, \mathbf{O}\right) \Phi^{\prime}(\mathbf{O}) \in \mathbb{Z}\left[G_{\phi^{m}} /\langle\phi\rangle\right] .
$$

The obvious quotient homomorphism takes $R\left(f^{m}, v\right)$ to $\bar{R}\left(f^{m}, v\right)$, the effect being to coalesce the distinct fixed point classes of $f^{m}$ which are in the same $m$-periodic orbit class of $f$, adding up the fixed point indices of the distinct pieces. The Lefschetz-Nielsen series of $f$ is the sequence $\left(\bar{R}\left(f^{m}, v\right)\right) \in \prod_{m>1} \mathbb{Z}\left[G_{\phi^{m}} /\langle\phi\rangle\right]$; we will see in Theorem 4.1 that the Lefschetz-Nielsen series is the "trace" of an interesting element of $K$-theory and that this fact implies the rationality of various zeta functions associated with the map $f$.

Let $\mathbb{Z} G^{\phi}[[t]]$ denote the $\phi$-twisted power series ring over $\mathbb{Z} G$, consisting of formal power series $\sum_{m>0} u_{m} t^{m}$ in the indeterminate $t$, with $u_{m} \in \mathbb{Z} G$, subject to the multiplication rule $t g=\bar{\phi}(g) t$ where $g \in G$. If $A$ is a square $\mathbb{Z} G$-matrix and $I$ is the identity matrix of the same size then the matrix $I-A t$ is invertible in the ring of $\mathbb{Z} G^{\phi}[[t]]$-matrices; its inverse is $\sum_{m>0}(A t)^{m}$. This simple observation turns out to be important. Define $\Delta(f, v) \in K_{1}\left(\mathbb{Z} G^{\phi}[[t]]\right)$ to be the element represented by the invertible matrix $c(f) \equiv$ $\prod_{k>0}\left(I-\tilde{f}_{k} t\right)^{(-1)^{k+1}}$. The matrix $c(f)$ depends on choices (of lifts, orientations etc., see $\S 2)$ but its torsion, $\Delta(f, v)$, does not $\left[\mathrm{GN}_{2}\right.$, Proposition 5.2]. 
We have a trace $\operatorname{Tr}_{1}(\Delta(f, v)) \in H H_{1}\left(\mathbb{Z} G^{\phi}[[t]]\right)$, but to obtain the Lefschetz-Nielsen series, we must compose this with a certain canonical homomorphism

$$
\left.P_{+}: H H_{1}\left(\mathbb{Z} G^{\phi}[[t]]\right)\right) \rightarrow \prod_{m \geq 1} \mathbb{Z}\left[G_{\phi^{m}} /\langle\phi\rangle\right]
$$

which we now define.

For $n \geq 0$, let $C_{1, n}$ be the abelian subgroup of $\mathbb{Z} G^{\phi}[[t]] \otimes \mathbb{Z} G^{\phi}[[t]]$ (the group of Hochschild 1-chains on $\left.\mathbb{Z} G^{\phi}[[t]]\right)$ generated by tensors of the form $y t^{a} \otimes z t^{b}$ where $y, z \in$ $\mathbb{Z} G$ and $a+b=n$, and let $C_{2, n}$ be the abelian subgroup of $\mathbb{Z} G^{\phi}[[t]] \otimes \mathbb{Z} G^{\phi}[[t]] \otimes \mathbb{Z} G^{\phi}[[t]]$ (the group of Hochschild 2-chains on $\left.\mathbb{Z} G^{\phi}[[t]]\right)$ generated by tensors of the form $u t^{a} \otimes v t^{b} \otimes w t^{c}$ where $u, v, w \in \mathbb{Z} G$ and $a+b+c=n$. Note that the Hochschild boundary operator $d_{2}$ (see $\S 3(\mathrm{~A}))$ maps $C_{2, n}$ into $C_{1, n}$. Define

$$
H H_{1, n}\left(\mathbb{Z} G^{\phi}[[t]]\right) \equiv \operatorname{ker}\left(d_{1}: C_{1, n} \rightarrow \mathbb{Z} G^{\phi}[[t]]\right) / \operatorname{image}\left(d_{2}: C_{2, n} \rightarrow C_{1, n}\right) .
$$

The homomorphism $\mathbb{Z} G^{\phi}[[t]] \otimes \mathbb{Z} G^{\phi}[[t]] \rightarrow \prod_{n=0}^{\infty} C_{1, n}$ given by

$$
\left(\sum_{i=0}^{\infty} y_{i} t^{i}\right) \otimes\left(\sum_{j=0}^{\infty} z_{j} t^{j}\right) \mapsto\left(\sum_{i+j=n} y_{i} t^{i} \otimes z_{j} t^{j}\right)_{n=0}^{\infty},
$$

where $y_{i}, z_{j} \in \mathbb{Z} G$, induces a homomorphism

$$
H H_{1}\left(\mathbb{Z} G^{\phi}[[t]]\right) \rightarrow \prod_{n \geq 0} H H_{1, n}\left(\mathbb{Z} G^{\phi}[[t]]\right) .
$$

Define a homomorphism $J_{n}: C_{1, n} \rightarrow \mathbb{Z} G$ as follows. Given a generating 1-chain $y t^{a} \otimes$ $z t^{b} \in C_{1, n}$, let $J_{n}\left(y t^{a} \otimes z t^{b}\right)=a y \phi^{a}(z)$. Suppose $\beta=u t^{a} \otimes v t^{b} \otimes w t^{c} \in C_{2, n}$. Then $J_{n}\left(d_{2} \beta\right)=b\left(v \phi^{b}(w) \phi^{b+c}(u)-u \phi^{a}(v) \phi^{a+b}(w)\right)$. Since $v \phi^{b}(w) \phi^{b+c}(u)-u \phi^{a}(v) \phi^{a+b}(w)$ maps to zero in $\mathbb{Z}\left[G_{\phi^{n}} /\langle\phi\rangle\right]$, it follows that $J_{n}$ induces a homomorphism:

$$
j_{n}: H H_{1, n}\left(\mathbb{Z} G^{\phi}[[t]]\right) \rightarrow \mathbb{Z}\left[G_{\phi^{n}} /\langle\phi\rangle\right] .
$$

Define $P_{+}: H H_{1}\left(\mathbb{Z} G^{\phi}[[t]]\right) \rightarrow \prod_{n=1}^{\infty} \mathbb{Z}\left[G_{\phi^{n}} /\langle\phi\rangle\right]$ to be the composite:

$$
\begin{aligned}
H H_{1}\left(\mathbb{Z} G^{\phi}[[t]]\right) \rightarrow \prod_{n \geq 0} H H_{1, n}\left(\mathbb{Z} G^{\phi}[[t]]\right) & \stackrel{\text { proj }}{\longrightarrow} \\
& \prod_{n \geq 1} H H_{1, n}\left(\mathbb{Z} G^{\phi}[[t]]\right) \\
& \stackrel{\prod_{n} j_{n}}{\longrightarrow} \prod_{n=1}^{\infty} \mathbb{Z}\left[G_{\phi^{n}} /\langle\phi\rangle\right] .
\end{aligned}
$$

The image of $\Delta(f, v)$ under $\operatorname{Tr}_{1}$ is the element of $H H_{1}\left(\mathbb{Z} G^{\phi}[[t]]\right)$ represented by the Hochschild 1-cycle:

$$
\sum_{k \geq 0}(-1)^{k+1} \sum_{i, j}\left(I-\tilde{f}_{k} t\right)_{i j} \otimes\left(\sum_{m \geq 0}\left(\tilde{f}_{k} t\right)^{m}\right)_{j i} .
$$

More importantly,

Theorem 4.1. The image of $\Delta(f, v)$ under

$$
P_{+} \circ \operatorname{Tr}_{1}: K_{1}\left(\mathbb{Z} G^{\phi}[[t]]\right) \rightarrow \prod_{m \geq 1} \mathbb{Z}\left[G_{\phi^{m}} /\langle\phi\rangle\right]
$$

is the Lefschetz-Nielsen series of $f,\left(\bar{R}\left(f^{m}, v\right)\right)$.

This is Theorem 5.6 of $\left[\mathrm{GN}_{2}\right]$. 
To relate this to zeta functions, consider a linear representation $\rho: G \rightarrow G L_{r}(S)$ where $S$ is a commutative ring with unit and $\rho \circ \phi=\rho$. The $\rho$-twisted Lefschetz number of $f$ is defined to be the image, $L(f, \rho)$, of $R(f, v)$ under the homomorphism induced by $\rho$. In detail, $\rho$ induces $\bar{\rho}: \mathbb{Z} G \rightarrow M_{r}(S)$, the ring of $r \times r$ matrices over $S$, and hence $\rho_{*} \equiv$ trace $\circ \bar{\rho}: \mathbb{Z} G \rightarrow S$. Define $L(f, \rho)=\sum_{k>0}(-1)^{k} \operatorname{trace}\left(\rho_{*}\left(\tilde{f}_{k}\right)\right) \in S$, where $\rho_{*}$ maps $\mathbb{Z} G$-matrices to $S$-matrices entry by entry. The homomorphism $\rho$ induces a function $G_{\phi} \rightarrow G L_{r}(S)$ because $\rho \circ \phi=\rho$, so $L(f, \rho)$ coalesces the components of

$$
R(f, v)=\sum_{\text {fixed point classes } \mathbf{F}} \iota(f, \mathbf{F}) \Phi(\mathbf{F})
$$

to give first an $S$-matrix, and then an element of $S$, namely the trace of that $S$-matrix.

The representation $\rho$ induces a homomorphism $K_{1}\left(\mathbb{Z} G^{\phi}[[t]]\right) \rightarrow K_{1}(S[[t]])$. The image of $\Delta(f, v)$ is $\Delta(f, \rho)$ represented by $c(f, \rho) \equiv \prod_{k>0}\left(I-\rho_{*}\left(\tilde{f}_{k}\right) t\right)^{(-1)^{k+1}}$. Applying the determinant homomorphism det $: K_{1}(S[[t]]) \rightarrow S[[\bar{t}]]$ we obtain

$$
\operatorname{det} \Delta(f, \rho)=\prod_{k \geq 0} \operatorname{det}\left(I-\rho_{*}\left(\tilde{f}_{k}\right) t\right)^{(-1)^{k+1}} \in S[[t]] .
$$

Define the $\rho$-twisted zeta function, $\zeta(f, \rho) \in S[[t]]$, to be $\exp \left(\sum_{m=1}^{\infty} \frac{1}{m} L\left(f^{m}, \rho\right) t^{m}\right)$. Then "rationality of the zeta function" is expressed by:

Theorem 4.2. $\operatorname{det} \Delta(f, \rho)=\zeta(f, \rho)$.

Theorem 4.2 is a direct consequence of Theorem 4.1. The homomorphism $\bar{\rho}$ induces

$$
\mathbb{Z}\left[G_{\phi^{m}} /\langle\phi\rangle\right] \rightarrow M_{r}(S) \stackrel{\text { trace }}{\longrightarrow} S
$$

which maps $\bar{R}\left(f^{m}, v\right)$ to $L\left(f^{m}, \rho\right)$; in fact, it induces a homomorphism

$$
K_{1}\left(\mathbb{Z} G^{\phi}[[t]]\right) \rightarrow \prod_{m \geq 1} \mathbb{Z}\left[G_{\phi^{m}} /\langle\phi\rangle\right] \rightarrow S[[t]]
$$

which maps $\Delta(f, v) \mapsto\left(\bar{R}\left(f^{m}, v\right)\right) \mapsto \zeta(f, \rho)$. Theorem 4.2 follows from the fact that this homomorphism equals $\Delta(f, v) \mapsto \Delta(f, \rho) \mapsto \operatorname{det} \Delta(f, \rho)$. The details can be found in $\left[\mathrm{GN}_{2}, \S 5(\mathrm{~B})\right]$.

Note that when $S=\mathbb{Z}, r=1$ and $\rho(G)=\{1\} \in \mathbb{Z}$ then $L(f, \rho)=L(f)$, the Lefschetz number of $f$, and $\zeta(f, \rho)$ is the familiar Lefschetz zeta function $\exp \left(\sum_{m=1}^{\infty} \frac{1}{m} L\left(f^{m}\right) t^{m}\right)$ of Milnor $[\mathrm{M}]$. Other choices give the zeta functions appearing in $\left[\mathrm{GN}_{2}\right],[\mathrm{J}]$, and $[\mathrm{F}]$.

In view of this, we regard the Lefschetz-Nielsen series as a "non-abelian" analogue of the zeta function. The invariant $\Delta(f, v)$ was introduced by us in $\left[\mathrm{GN}_{2}\right]$ and is an example of Fact (6) in the Introduction. We discovered it through our study of $\operatorname{Tr}_{1}(\Delta(f, v))$. This is explained in $\left[\mathrm{GN}_{2}\right]$, where we also show that $\Delta(f, v)$ depends only on the homotopy class of $f$ and on the simple homotopy type of $K$.

\section{First-order Euler characteristics}

5(A). More on one-parameter Nielsen fixed point theory. Let $F: K \times I \rightarrow K$ be a cellular homotopy where $K$ is a finite CW complex and $F_{0}=F_{1}=\mathrm{id}_{K}$. Just as in $\S 3(\mathrm{~A})$, we set $G \equiv \pi_{1}(K, v)$ and identify $G$ with $\pi_{1}(K \times I,(v, 0))$; here, $v$ is a vertex of $K$. And just as in $\S 2$, we consider the cellular chains $C_{*}(\tilde{K})$ and $C(K): F$ induces a chain homotopy 
$\left\{D_{k}: C_{k}(K) \rightarrow C_{k+1}(K)\right\}$, and $\tilde{F}$ induces a chain homotopy $\left\{\tilde{D}_{k}: C_{k}(\tilde{K}) \rightarrow C_{k+1}(\tilde{K})\right\}$. Precisely: $\tilde{D}_{k}(\tilde{e})=(-1)^{k+1} \tilde{F}_{k}(\tilde{e} \times I)$ and $D_{k}(e)=(-1)^{k+1} F_{k}(e \times I)$ where $e$ is any $k$-cell of $K$ (with a chosen orientation as in $\S 2$ ). ${ }^{2}$

We also have the boundary maps $\left\{\partial_{k}: C_{k}(K) \rightarrow C_{k-1}(K)\right\}$ and $\left\{\tilde{\partial}_{k}: C_{k}(\tilde{K}) \rightarrow\right.$ $\left.C_{k-1}(\tilde{K})\right\}$. If $g_{0} \in G$ is represented by the loop $F(\{v\} \times I)$ then $\tilde{F}_{1}(\tilde{e})=\tilde{e} g_{0}^{-1}$; here $g_{0}^{-1}$ appears rather than $g_{0}$ because the natural left action of $G$ has been turned into a right action.

The chain homotopy relations give matrix equations:

$$
\tilde{D}_{k-1} \tilde{\partial}_{k}+\tilde{\partial}_{k+1} \tilde{D}_{k}=I\left(1-g_{0}^{-1}\right) \quad \text { and } \quad D_{k-1} \partial_{k}+\partial_{k+1} D_{k}=0
$$

where $I$ is a suitable identity matrix.

It is convenient to write $\tilde{D} \equiv \bigoplus_{k}(-1)^{k+1} \tilde{D}_{k}$ and $\tilde{\partial} \equiv \bigoplus_{k} \tilde{\partial}_{k}$, with similar definitions for $D$ and $\partial$. Then

$$
\operatorname{trace}(\tilde{D} \tilde{\partial}-\tilde{\partial} \tilde{D})=\left(1-g_{0}^{-1}\right) \chi(K) \quad \text { and } \quad D \partial-\partial D=0
$$

where $\chi(K)$ is the Euler characteristic of $K$.

Proposition 5.1. The Hochschild 1-chain $\sum_{i, j} \tilde{\partial}_{i j} \otimes \tilde{D}_{j i}$ is a cycle.

Proof. (Compare $\left[\mathrm{GN}_{3}\right.$, Proposition 2.4].) The image of $\sum_{i, j} \tilde{\partial}_{i j} \otimes \tilde{D}_{j i}$ under the Hochschild boundary $\left(d_{1}\right.$ of $\left.\S 3(\mathrm{~A})\right)$ is $\left(1-g_{0}^{-1}\right) \chi(K)$. If $\chi(K)=0$ the conclusion follows. At any rate $\left(1-g_{0}^{-1}\right) \chi(K)$ is a boundary, so it represents zero in $H H_{0}(\mathbb{Z} G)$, and hence if $\chi(K) \neq 0$ we must have $g_{0}^{-1}=1$.

5(B). First-order Euler characteristics. Let $\Gamma_{K} \equiv \pi_{1}\left(K^{K}\right.$,id). Thus $\gamma \in \Gamma_{K}$ is represented by $F^{\gamma}: K \times I \rightarrow K$ as in (A). We write $\tilde{D}^{\gamma}$ and $D^{\gamma}$ for the corresponding chain homotopies. By Proposition 5.1 we may define a function $\tilde{\mathcal{X}}_{1}(K): \Gamma_{K} \rightarrow H H_{1}(\mathbb{Z} G)$ by

$$
\tilde{\mathcal{X}}_{1}(K)(\gamma)=\text { homology class of } \sum_{i, j} \tilde{\partial}_{i j} \otimes \tilde{D}_{j i}^{\gamma} \text {. }
$$

There is a left action of $\Gamma_{K}$ on $H H_{1}(\mathbb{Z} G)$ given at the level of chains by $\gamma\left(g_{1} \otimes g_{2}\right)=$ $g_{1} \otimes g_{2} \eta(\gamma)^{-1}$; here, $g_{1}, g_{2} \in G$ and $\eta: \Gamma_{K} \rightarrow G$ takes $\gamma$ to the element of $G$ represented by the loop $F^{\gamma}(v, \cdot)$. The image of $\eta$ is called the Gottlieb subgroup of $\pi_{1}(K, v)$ and lies in the center, $Z(G)$, of $G$.

Thus we may regard $H H_{1}(\mathbb{Z} G)$ as a left $\Gamma_{K}$-module, and it is easy to see that:

Proposition 5.2. The function $\tilde{\mathcal{X}}_{1}(K)$ is a derivation, i.e.,

$$
\tilde{\mathcal{X}}_{1}(K)\left(\gamma_{1} \gamma_{2}\right)=\tilde{\mathcal{X}}_{1}(K)\left(\gamma_{1}\right)+\gamma_{1} \tilde{\mathcal{X}}_{1}(K)\left(\gamma_{2}\right)
$$

for all $\gamma_{1}, \gamma_{2} \in \Gamma_{K}$.

If $M$ is a left $\Gamma_{K}$-module then the cohomology group $H^{1}\left(\Gamma_{K}, M\right)$ is naturally identified with the quotient, $\operatorname{Der}\left(\Gamma_{K}, M\right) / \operatorname{Inn}\left(\Gamma_{K}, M\right)$, of derivations modulo inner derivations

\footnotetext{
2 We use the following sign convention. If $\tilde{e}$ is an oriented $k$-cell of $\tilde{K}$ then $\tilde{D}_{k}(\tilde{e})$ is the $(k+1)$-chain $(-1)^{k+1} \tilde{F}_{*}(\tilde{e} \times I) \in C_{k+1}(\tilde{K})$, where $\tilde{e} \times I$ is given the product orientation. This is consistent with the convention that if $E_{i, \epsilon}$ is the face of the cube $I^{n}=[0,1]^{n}$ obtained by holding the $i^{\text {th }}$ coordinate fixed at $\epsilon=0$ or 1 , then the incidence number $\left[I^{n}: E_{i, \epsilon}\right]$ is $(-1)^{i+\epsilon}$. At the level of cellular $n$-chains, we have $\partial_{n} I^{n}=\sum_{i, \epsilon}\left[I^{n}: E_{i, \epsilon}\right] E_{i, \epsilon}$.
} 
(recall that a derivation $\Gamma_{K} \rightarrow M$ is inner if it is of the form $\gamma \mapsto(1-\gamma) m$ for some $m \in M)$. We denote the image of a derivation $\Theta: \Gamma_{K} \rightarrow M$ in $H^{1}\left(\Gamma_{K}, M\right)$ by $[\Theta]$.

The enhanced first-order Euler characteristic of $K$ is the cohomology class:

$$
\tilde{\chi}_{1}(K) \equiv\left[\tilde{\mathcal{X}}_{1}(K)\right] \in H^{1}\left(\Gamma_{K}, H H_{1}(\mathbb{Z} G)\right) .
$$

To explain why we call this invariant "enhanced" we digress to define a simpler invariant with which $\tilde{\chi}_{1}(K)$ should be compared. The homology of $G$ with integer coefficients can be computed from a chain complex which in degrees $2,1,0$ is:

$$
\cdots \rightarrow \mathbb{Z} G \otimes \mathbb{Z} G \stackrel{d_{2}^{\prime}}{\longrightarrow} \mathbb{Z} G \stackrel{d_{1}^{\prime}}{\longrightarrow} \mathbb{Z}
$$

where $d_{2}^{\prime}\left(g_{1} \otimes g_{2}\right)=g_{2}-g_{1} g_{2}+g_{1}$ for $g_{1}, g_{2} \in G$ and $d_{1}^{\prime}(g)=1$ for $g \in G$. [This is obtained from the Hochschild complex of $\S 3(\mathrm{~A})$ by replacing the rightmost tensor factor of $\mathbb{Z} G$ by $\mathbb{Z}$, i.e. $H_{*}(G) \cong H H_{*}(\mathbb{Z} G, \mathbb{Z})$ where $H H_{*}(\mathbb{Z} G, \mathbb{Z})$ is Hochschild homology with coefficients in the trivial bimodule $\mathbb{Z}$.] Let $A: \mathbb{Z} G \rightarrow G_{\text {ab }}$ extend the "abelianization" homomorphism $G \rightarrow G_{\mathrm{ab}} \equiv H_{1}(G)$. There is a natural homomorphism $\varepsilon: H H_{1}(\mathbb{Z} G) \rightarrow H_{1}(G)$ defined by $\varepsilon\left(\left\{\sum_{i} n_{i} g_{1, i} \otimes g_{2, i}\right\}\right)=A\left(\sum_{i} n_{i} g_{1, i}\right)$ where $\sum_{i} n_{i} g_{1, i} \otimes g_{2, i}$ is a Hochschild cycle and $\{\cdot\}$ denotes Hochschild homology class. The homomorphism $\varepsilon$ induces:

$$
\varepsilon_{*}: H^{1}\left(\Gamma_{K}, H H_{1}(\mathbb{Z} G)\right) \rightarrow H^{1}\left(\Gamma_{K}, H_{1}(G)\right) \cong \operatorname{Hom}\left(\Gamma_{K}, H_{1}(G)\right) .
$$

In $\left[\mathrm{GN}_{3}\right]$ we defined the first-order Euler characteristic of $K$ to be the homomorphism $\chi_{1}(K): \Gamma_{K} \rightarrow H_{1}(G)$ given by $\chi_{1}(K)=A\left(\sum_{i, j} \tilde{\partial}_{i j} D_{j i}\right)$. The comparison with $\tilde{\chi}_{1}(K)$ is:

Proposition 5.3. $\varepsilon_{*}\left(\tilde{\chi}_{1}(K)\right)=\chi_{1}(K)$.

These first order Euler characteristics appear to be useful new invariants. Applications of $\chi_{1}(K)$ to group theory are given in $\left[\mathrm{GN}_{3}\right]$ and to geometry in [GNO], while applications of $\tilde{\chi}_{1}(K)$ to differential topology and to 3 -dimensional Seifert fibered spaces are given in $\left[\mathrm{GN}_{4}\right]$.

REMARK. The invariant $\chi_{1}(K)$ is a natural object from the point of view of algebraic topology; over a field of coefficients $\mathbb{F}, \chi_{1}(K ; \mathbb{F})$ can be expressed homologically in terms of cap (or cup) products (see $\left.\left[\mathrm{GN}_{3}\right]\right)$ and higher analogs $\chi_{n}(K ; \mathbb{F}), n>1$ can be defined and calculated [GNO].

5(C). The trace. Here is a simple observation (see $\left[\mathrm{GN}_{4}\right.$, Proposition 1.4]):

Proposition 5.4. The image of the composite homomorphism:

$$
\pm G \rightarrow K_{1}(\mathbb{Z} G) \stackrel{\operatorname{Tr}_{1}}{\longrightarrow} H H_{1}(\mathbb{Z} G)
$$

lies in the component of $H H_{1}(\mathbb{Z} G) \cong \bigoplus_{C \in G_{1}} H_{1}\left(Z\left(g_{C}\right)\right)$ corresponding to the trivial conjugacy class [1].

Proposition 5.4 implies that when $\mathrm{Wh}(G)=0$ the image of $\operatorname{Tr}_{1}: K_{1}(\mathbb{Z} G) \rightarrow H H_{1}(\mathbb{Z} G)$ is concentrated in the component corresponding to the trivial conjugacy class. On the other hand, in $\left[\mathrm{GN}_{4}, \S 6\right]$ we give examples of finite complexes $K$ which have the following properties:

(1) $K$ triangulates a closed aspherical 3-manifold.

(2) $\mathrm{Wh}(G)=0$. 
(3) $K$ admits the structure of an oriented Seifert fibered space and the element $\gamma \in \Gamma_{K}$ given by the $S^{1}$-action on $K$ associated to this Seifert fibered space structure is such that $\tilde{\mathcal{X}}_{1}(K)(\gamma)$ is not concentrated in the component of the trivial conjugacy class.

Moreover, in these examples the third property persists after passage to the cohomology class $\tilde{\chi}_{1}(K)$. More precisely, $\operatorname{Tr}_{1}: K_{1}(\mathbb{Z} G) \rightarrow H H_{1}(\mathbb{Z} G)$ extends to a $\Gamma_{K}$-module homomorphism $\operatorname{Tr}_{1}^{\prime}: \mathbb{Z} \Gamma_{K} \otimes K_{1}(\mathbb{Z} G) \rightarrow H H_{1}(\mathbb{Z} G)$ and $\tilde{\chi}_{1}(K)$ is not in the image of the induced homomorphism $\left(\operatorname{Tr}_{1}^{\prime}\right)_{*}: H^{1}\left(\Gamma_{K}, \mathbb{Z} \Gamma_{K} \otimes K_{1}(\mathbb{Z} G)\right) \rightarrow H^{1}\left(\Gamma_{K}, H H_{1}(\mathbb{Z} G)\right)$.

\section{References}

[Ba] H. BAss, Euler characteristics and characters of discrete groups, Invent. Math. 35 (1976), 155-196.

[B] R. F. Brown, The Lefschetz Fixed Point Theorem, Scott Foresman, Chicago, 1971.

[Br] K. S. Brown, Cohomology of Groups, Springer-Verlag, New York, 1982.

[BG] K. S. Brown and R. Geoghegan, An infinite dimensional torsion-free $F P_{\infty}$ group, Invent. Math. 77 (1984), 367-381.

[C] M. M. Cohen, A Course in Simple-Homotopy Theory, Springer-Verlag, New York, 1973.

[DG] D. Dimovski and R. Geoghegan, One-parameter fixed point theory, Forum Math. 2 (1990), 125-154.

[F] D. FriED, Homological identities for closed orbits, Invent. Math. 71 (1983), 419-442.

$\left[\mathrm{G}_{1}\right]$ R. Geoghegan, Fixed points in finitely dominated compacta: the geometric meaning of a conjecture of H. Bass, in: Shape Theory and Geometric Topology, Lecture Notes in Math. 870, Springer-Verlag, New York, 1981, 6-22.

$\left[\mathrm{G}_{2}\right]$ R. Geoghegan, Nielsen fixed point theory, in: Handbook of Geometric Topology, (to be published by Elsevier).

$\left[\mathrm{GN}_{1}\right]$ R. Geoghegan and A. Nicas, Parametrized Lefschet $z-N i e l s e n$ fixed point theory and Hochschild homology traces, Amer. J. Math. 116 (1994), 397-446.

$\left[\mathrm{GN}_{2}\right]$ R. Geoghegan and A. Nicas, Trace and torsion in the theory of flows, Topology 33 (1994), 683-719.

[GN 3 R. Geoghegan and A. Nicas, Higher Euler characteristics (I), Enseign. Math. 41 (1995), 3-62.

$\left[\mathrm{GN}_{4}\right]$ R. GEOGhegan and A. NicAs, A Hochschild homology Euler characteristic for circle actions, $K$-theory (to appear).

[GNO] R. Geoghegan, A. Nicas and J. Oprea, Higher Lefschetz traces and spherical Euler characteristics, Trans. Amer. Math. Soc. 348 (1996), 2039-2062.

[HH] H. M. Hastings and A. Heller, Homotopy idempotents on finite dimensional complexes split, Proc. Amer. Math. Soc. 85 (1982), 619-622.

[J] B. J. JIANG, Estimation of the number of periodic orbits, Pacific J. Math. 172 (1996), 151-185.

[K] M. A. Kervaire, Le théorème de Barden-Mazur-Stallings, Comment. Math. Helv. 40 (1965), 31-42.

[M] J. Milnor, Infinite cyclic coverings, in: Conference on the Topology of Manifolds (Michigan State Univ., E. Lansing, Mich., 1967), Prindle, Weber \& Schmidt, Boston, 1968, 115-133. 
[R] K. Reidemeister, Automorphismen von Homotopiekettenringen, Math. Ann. 112 (1936), 586-593.

[RS] C. P. Rourke and B. J. SAnderson, Introduction to Piecewise-Linear Topology, Springer-Verlag, New York, 1972.

[St] J. Stallings, Centerless groups-An algebraic formulation of Gottlieb's theorem, Topology 4 (1965), 129-134.

[Wa] C. T. C. WALl, Finiteness conditions for CW complexes, Ann. of Math. 81 (1965), 56-69.

[W] F. Wecken, Fixpunktklassen, I, II, III, Math. Ann. 117 (1941), 659-671, 118 (1942), 216-234, 118 (1942), 544-577. 